\section{Poster child}

W

e regret the human cost to those coping with the effects of mutations, but we gratefully accept the responsibility to publish pictures of identifiable individuals living with genetic disorders. For this practice to serve both its humane and its educational purposes, we require informed consent from the individual depicted, specifying that we have permission to publish his or her photograph. For minors or individuals who are unable to give consent, we require consent from a parent or legal guardian. Obscuring part of a person's photograph for anonymity is not, in our view, an adequate solution, because the ethical problem of accurately portraying a whole person has not been addressed and the whole story of the disorder has not been told. We do not think that research participants are humanely represented with black bars over their eyes, nor is this the best practice with which to undertake the genetic screen of the human population.

Ethical review of human genetics research normally requires that researchers obtain consent to use photographs of human participants in scientific presentations. We maintain that publication requires further consideration because its permanence makes it qualitatively different from a research talk. We publish a journal of record, and articles in print and on the website have a long useful life and are accessible to both specialist and nonspecialist readers. We therefore recommend obtaining specific consent to depict a living individual whose life might be affected by publication of his or her picture. We do not seek specific consent to publish other photographs supplied by authors, provided that these are obtained in accordance with the stipulations of the relevant ethical review board. We do, however, urge ethicists to consider publication as a distinct research activity to which families segregating genetic disorders make a substantial contribution.

Showing the faces of individuals with developmental syndromes and other genetic diseases in the journal serves two necessary functions. First, the individuals are recognized as participants in the research, without whom these discoveries would not have been made. Second, presenting all the features of an affected individual, both normal and malformed, alerts and educates readers, especially clinicians and geneticists, with more than a mere checklist of affected parts. Rather, we hope to make use of one of the most sensitive tools in the human genetic screen: the remarkable ability of humans to recognize one another, a skill which may be open to calibration, at least in the case of face recognition (e.g., Nature 428, 557-561; 2004). Currently, the most obvious ways in which individuals with a particular syndrome resemble one another can be used in diagnosis without noting departure from the population range of familial resemblance. In the future, it may be possible for dysmorphologists to discern more subtle developmental changes as typical of particular syndromes, against a better characterized background of population variation.

Full phenotypic description unquestionably enables research, because phenotypic features peripheral to one study may be central to the next. Syndromes fall into phenotypic families, and the uses and limitations of phenotypic overlap in uncovering shared functions has been recently reviewed (Nat. Rev. Genet. 5, 545-551; 2004). For example, Emma Tonkin and colleagues (Nat. Genet. 36, 636-641; 2004) speculated that growth delay and limb reduction phenotypes shared between Cornelia de Lange syndrome and Roberts syndrome reflect related molecular causes. Now, on page 468 of this issue, Hugo Vega and colleagues describe mutations underlying a sister chromatid cohesion defect in Roberts syndrome. Taken together, these papers suggest that chromatid cohesion defects contribute to a partially overlapping spectrum of anomalies in the two syndromes. Whether enhancer-promoter interactions (Ian Krantz and colleagues, Nat. Genet. 36, 631-635; 2004) are involved in the two pathologies remains to be studied.

Research, though it supplies hope and explanation, is only part of the solution to human disease. Compassion, promoting understanding and providing care are still the key factors in coping with genetic disorders. It is quite humbling to compare the papers we publish with some of the websites organized around the lives of people, many of them children, who live with the consequences of deleterious gene variants. These each highlight the life of a person and summon support, recognition and research. With appropriate ethical consideration, a research publication can attempt to do the same. 